\title{
Synthesis and Characterization of Ni Doped ZnO Nanoparticles
}

\author{
M.R.A. Bhuiyan, ${ }^{\mathrm{a},}$, M.K. Rahman ${ }^{\mathrm{a}}$ \\ ${ }^{a}$ Department of Applied Physics, Electronics and Communication Engineering, Islamic University, \\ Kushtia-7003, Bangladesh
}

\begin{abstract}
This paper discerns key ideas and themes of the possibility of growing $\mathrm{Ni}$ doped $\mathrm{ZnO}$ nanoparticles by electrochemical method. The purpose is to study the growth mechanism and to optimize the parameters of this method. Upon successful synthesizing the samples, they were characterized using various techniques. XRD, SEM, FTIR, photoluminescence spectroscopy together with the measured optical parameters obtained from UV-VIS absorption testing were analyzed. The X-ray diffraction (XRD) was measured by using a Bruker D8 Advance X-ray diffractometer with $\mathrm{CuK}_{\alpha}$ radiation. The surface morphology was investigated using an 'EVO LS 15' scanning electron microscope. The FTIR absorption spectra were recorded on a Perkin-Elmer GX FTIR system. The PL spectra were collected on a Jobin Yvon-Horiba Triax 190 spectrometer with a spectral resolution of $0.3 \mathrm{~nm}$. UV-VIS absorption spectrum was recorded by using a UV-VIS spectrophotometer in the photon wavelength range between 300 and $600 \mathrm{~nm}$. XRD pattern reveals that the polycrystalline of hexagonal wurtzite structure and the average size of the particles were estimated to be approximately $61 \mathrm{~nm}$, which conform the nanoparticle. The FTIR result shows the stretching vibration of the $\mathrm{Zn}-\mathrm{O}$ bond in Ni doped $\mathrm{ZnO}$ nanoparticles. There is a green emission peak centered at about $384 \mathrm{~nm}$ in the PL behavior. The band edge is shifted to the lower energy side of the Ni doped $\mathrm{ZnO}$ nanoparticle. Analyzing the results of various types of characterizations, it has been assessed that $\mathrm{Ni}$ doped $\mathrm{ZnO}$ nanoparticles was successfully synthesized.
\end{abstract}

Index Terms: Ni doped $\mathrm{ZnO}$, Compositional uniformity, Structural parameter, Optical parameter.

(C) 2014. Published by MECS Publisher. Selection and/or peer review under responsibility of the Research Association of Modern Education and Computer Science.

\section{Introduction}

Nanometer-sized particles $(1-100 \mathrm{~nm})$ have attracted considerable interest for a wide variety of applications, ranging from electronics via ceramics to catalysts due to their unique or improved properties, which are primarily determined by size, composition, and structure [1]. These properties are strongly related to the synthesis processes. Numerous solution techniques, for example, sol-gel, emulsion, colloidal, and

* Corresponding author. Tel.:+88 01711 443079; fax: +88 07162399

E-mail address: mrab_iu@yahoo.com 
aerosol processes, have been used to synthesize a variety of nanoparticles [2-6]. As an alternative approach, the electrochemical route is of considerable interest because of a possibly precise particle size control achieved by adjusting current density or applied potential. For electrochemical synthesis, extensive investigations have been focused on the metal particles, especially on noble metal particles. This method, in fact, skillfully combines an electrochemical process with plasma at ambient pressure and temperature. Compared with other methods of synthesizing $\mathrm{Ni}$ doped $\mathrm{ZnO}$ powder, this access has some advantages. Firstly, the whole process, with a simple experimental set-up and electrolyte system is performed under mild conditions. Secondly, particles can be obtained within a few minutes. Finally, changing the volume ratio of the electrolyte can effectively control the size of the obtained particles. The earlier study of the magnetic nature of $\mathrm{Ni}$ doped $\mathrm{ZnO}$ shows different results. Some authors [7, 8] reported that, $\mathrm{ZnO}$ doped with 5 to 25at.\% Ni did not show room temperature ferromagnetic (RT-FM) behavior and the FM characteristics disappeared for temperatures higher than 30K. Wakano et al. [9] observed and measured FM properties in 3 to 25 at.\% $\mathrm{Ni}$ doped $\mathrm{ZnO}$ at temperatures less than $30 \mathrm{~K}$, however, super paramagnetic behavior was observed at least up to 300K. i.e. absent of RT-FM. Liu et al. [10] have noticed that the preparation details of the prepared $\mathrm{Ni}$ doped $\mathrm{ZnO}$ has great influence on their magnetic properties, so that annealing at $800 \mathrm{oC}$ in $\mathrm{Ar}$ gas atmosphere significantly increases the magnetization, however, RT-FM was not detected. Recently, the RTFM was observed in $~ 12$ at. $\% \mathrm{Ni}$ doped $\mathrm{ZnO}$ prepared by sputtering method [11] and in 5at.\% Ni doped $\mathrm{ZnO}$ prepared by laser ablation method on sapphire substrate [12]. Thus, the experimental evidence for the existence of RT-FM phase in $\mathrm{Ni}$ doped $\mathrm{ZnO}$ at room temperature is still questionable and needs more investigations. Generally, experiments show that the magnetization properties of $\mathrm{ZnO}$ :Ni depend strongly on the preparation method and procedure and seems to be sensitive to the crystalline structure and concentration of intrinsic (like oxygen vacancies) and extrinsic defects. A clear understanding of the RT-FM ordering can provide us with new experimental approaches to an opportunity to develop spintronic devices based on $\mathrm{Ni}$ doped $\mathrm{ZnO}$.

\section{Experimental Details}

\subsection{Synthesis of Ni doped ZnO Nanoparticles}

The synthesis method has been employed a modified version of the originally used by Reetz and Helbig [13] for metal particles (in a one-phase electrochemical system). Ni doped $\mathrm{ZnO}$ particles were synthesized at room temperature by an electrochemical route. The electrolytic bath consisted of acetonitrile and tetrahydrofuran (THF) mixed in the ratio $4: 1$, in which high purity $\mathrm{Ni}$ doped $\mathrm{Zn}$ metal sheet $(1 \mathrm{~cm} \times 1 \mathrm{~cm})$ and laboratory grade platinum sheet $(1 \mathrm{~cm} \times 1 \mathrm{~cm})$ served as anode and cathode respectively. The capping agent tetra-trimethyle ammonium-bromide (TTAB) also served as the electrolyte. Electrolysis was carried out in nitrogen atmosphere for a few hours in constant current mode (GPS-30D, 0-30V, 0-5A). Current density was maintained to obtain different sized of particles. The molarity of TTAB in the chemical bath was varied from $0.1 \mathrm{mM}$ to $0.9 \mathrm{mM}$. The white $\mathrm{Ni}$ doped $\mathrm{ZnO}$ particles remain suspended in the solvent and separated by using centrifugation. On drying, a free flowing powder of $\mathrm{Ni}$ doped $\mathrm{ZnO}$ particles are obtained.

\subsection{Measurements}

The structural properties of the $\mathrm{Ni}$ doped $\mathrm{ZnO}$ particles were measured by using a Bruker D8 Advance Xray diffractometer with $\mathrm{CuK} \alpha$ radiation of wavelength $\lambda=1.54056 \AA$. The X-ray diffraction (XRD) measurements were carried out in the locked coupled mode in the $2 \theta$ range of 20 to $60 \mathrm{o}$. The surface morphology and composition of $\mathrm{Ni}$ doped $\mathrm{ZnO}$ particles were investigated by using an 'EVO LS 15' scanning electron microscope developed by Carl Zeiss. An accelerating voltage of 15 to $19 \mathrm{keV}$ and probe current of >00 pA. UV-VIS absorption spectrum of $\mathrm{Ni}$ doped $\mathrm{ZnO}$ particles were recorded by using a UV-VIS 
spectrophotometer in the photon wavelength range between 300 and $600 \mathrm{~nm}$. The FTIR absorption spectra were recorded on a Perkin-Elmer GX FTIR system used to obtain $16 \mathrm{~cm}-1$ resolution spectra in the range 500 to $4000 \mathrm{~cm}-1$ region, scanned 30 times (absorbance mode), in order to exploit the instrumental built-up noise reduction algorithm. The Photoluminescence (PL) measurements were carried out from room temperature by employing a $488 \mathrm{~nm}$ line of an argon ion laser. The PL spectra were collected on a Jobin Yvon-Horiba Triax 190 spectrometer with a spectral resolution of $0.3 \mathrm{~nm}$, coupled with a liquid nitrogen-cooled CCD detector.

\section{Results and Discussion}

\subsection{Structural and Compositional}

X-ray diffraction (XRD) is mainly used for phase identification. Fig.1 shows the XRD pattern of the prepared Ni-doped $\mathrm{ZnO}$ sample. The grown sample shows the peaks of (100) and (101). No signals of the metallic $\mathrm{Zn}$ are detected by XRD. Also, there is no peak corresponding with the $\mathrm{Ni}$, suggesting that the $\mathrm{Ni}$ element may be doped into $\mathrm{ZnO}$. This pattern reveals that the polycrystalline of hexagonal wurtzite structure that is known $\mathrm{ZnO}$ structure. The Bragg angle of the intense (101) reflection is observed as light shift towards higher values relative to that of pure $\mathrm{ZnO}$ which has been indicated that $\mathrm{Ni}$-doped $\mathrm{ZnO}$ was formed along with $\mathrm{NiO}$ phase. Of course, this happens relating to the limit of the solids solubility of $\mathrm{Ni}$ in $\mathrm{ZnO}$. This is an evidence for creation of internal compressive micro stress. Such case was also observed in $\mathrm{ZnO}$ annealed in hydrogen atom sphere [14]. It is known that more oxygen content is introduced into the sample. It is clear that the (101) peak is sharper and stronger.

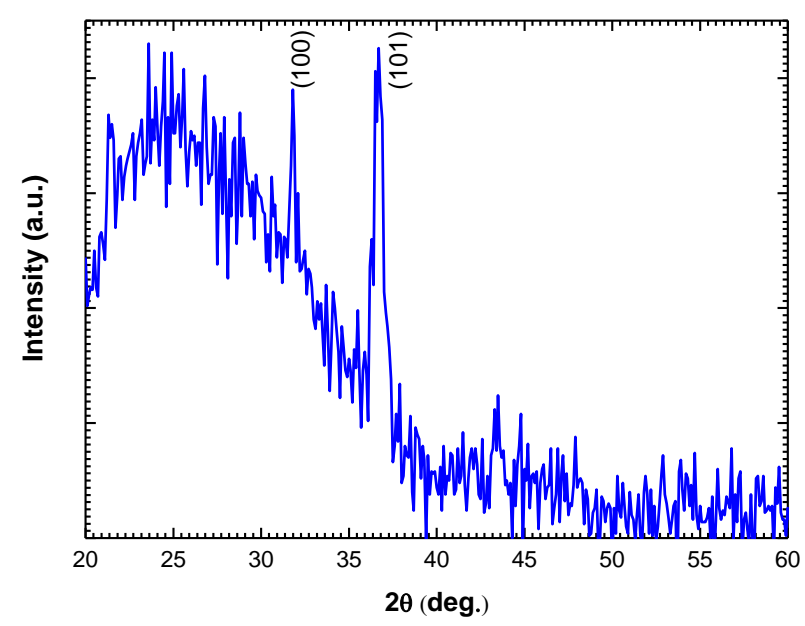

Fig. 1. XRD spectrum of $\mathrm{Ni}$ doped $\mathrm{ZnO}$ particles

The size of the Ni doped $\mathrm{ZnO}$ particles was estimated by applying the Scherrer equation [15] to the half intensity width of the (101) peak:

$$
\mathrm{d}=\frac{\mathrm{k} \lambda}{\beta \cos \theta}
$$


where $\mathrm{k}$ is the particle shape factor and taken as 0.827 because of the hexagonal Ni doped $\mathrm{ZnO}$ particles, $\lambda$ is the wavelength of $\mathrm{CuK} \alpha$ radiation $(0.154 \mathrm{~nm}), \beta$ is the calibrated half intensity width of the selected diffraction peak (degrees), and $\theta$ is the Bragg angle (half of the peak position angle). From this equation, the average size of the $\mathrm{Ni}$ doped $\mathrm{ZnO}$ particles was estimated to be approximately $61 \mathrm{~nm}$, which conforms reasonably well to the literature value [16]. This implies that the samples are successfully synthesized.

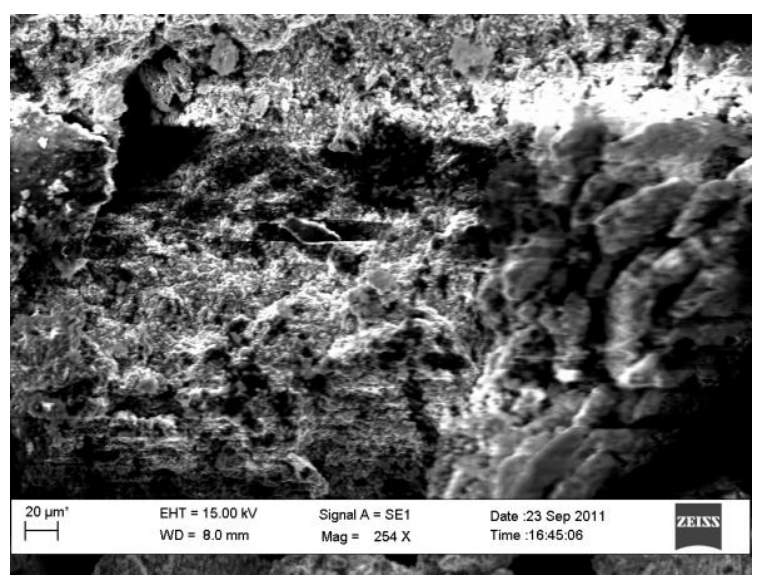

Fig. 2. SEM image of Ni doped $\mathrm{ZnO}$ particles

SEM image in fig. 2 shows the morphology of $\mathrm{Ni}$ doped $\mathrm{ZnO}$ particles. It is observed that there is a rough surface in the particles.

\subsection{Optical absorption}

Substation of Ni cations in tetrahedral sites of the wurtzite structure was further conformed by UV-vis optical spectroscopy. The room temperature spectra of the $\mathrm{Ni}$ doped $\mathrm{ZnO}$ particles are reported in fig. 3 and compared to the spectrum recorded for the other researcher report [17].

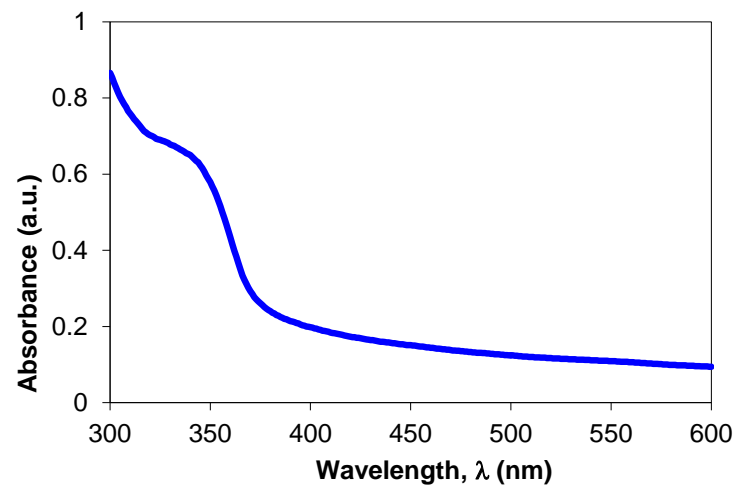

Fig. 3. Optical absorption spectrum of $\mathrm{Ni}$ doped $\mathrm{ZnO}$ particles 
From this figure, the band edge is shifted to the lower energy side of the $\mathrm{Ni}$ doped $\mathrm{ZnO}$ samples. The decrease in the band edge is a clear indication for the incorporation of $\mathrm{Ni}$ inside the $\mathrm{ZnO}$ lattice [18].

\subsection{FTIR absorption}

To make the clear interactions with the particles, FTIR absorption spectrum of the particles was performed. Fig. 4 shows the FTIR spectrum of Ni doped $\mathrm{ZnO}$ particles. The peak at $3180 \mathrm{~cm}-1$ is the stretching vibration of the H-O bond. The peaks at 1620 and $1400 \mathrm{~cm}-1$ are assigned to the vibrations of amide I and amide II, respectively. The peaks are similar to other worker [19]. A peak at $530 \mathrm{~cm}-1$ is the stretching vibration of the $\mathrm{Zn}-\mathrm{O}$ bond in doped $\mathrm{ZnO}$ particles.

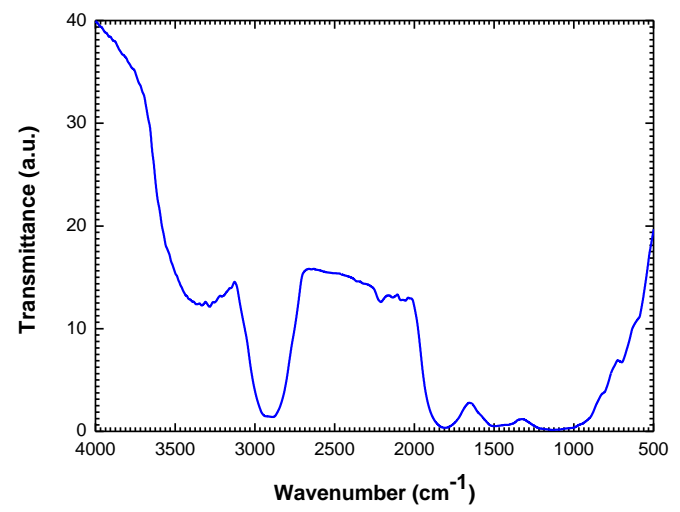

Fig. 4. FTIR spectrum of Ni doped $\mathrm{ZnO}$ particles

\subsection{Photoluminescence spectroscopy}

The room-temperature PL spectrum on the Ni doped $\mathrm{ZnO}$ sample is shown in fig. 5. Only a UV emission

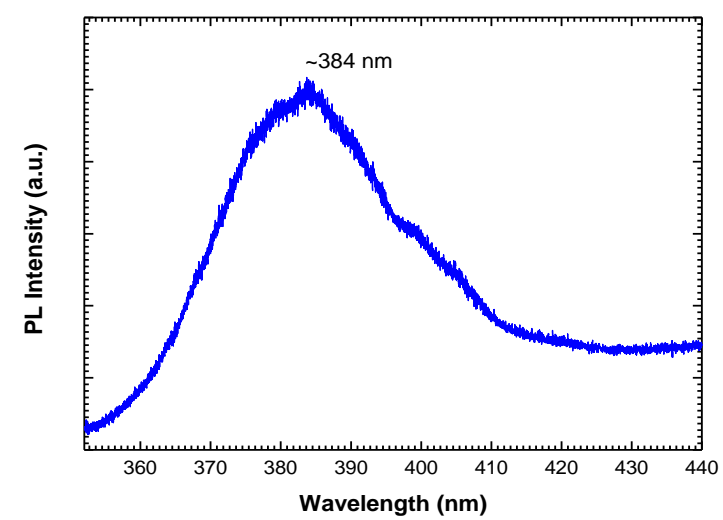

Fig. 5. Photoluminescence spectrum of Ni doped $\mathrm{ZnO}$ particles 
peak can be observed on the pure $\mathrm{ZnO}$ cone arrays without any significant emission in visible region, though the green emission is often observed in many reports [20-23]. The UV emission is attributed to the near-bandedge emission from the recombination of free excitons through an exciton-exciton collision process [24]. Vanheusden et al. [22] proved that the individually ionized oxygen vacancy is responsible for the green emission in $\mathrm{ZnO}$. To observe the green emission that indicates its good crystallization with little oxygen vacancy. After the Ni ion incorporation, the UV emission peak shifts to a little longer wavelength, consistent with the result of the UV-visible absorption. Besides, the intensity of UV peaks of Ni doped ZnO decreases. There is a green emission peak centered at about $384 \mathrm{~nm}$. As more oxygen ions would be pulled out from lattices by $\mathrm{Ni}$ ions to form $\mathrm{NiO}$ in interstices of lattices.

\section{Conclusions}

In summary, $\mathrm{Ni}$ doped $\mathrm{ZnO}$ particles have been synthesized by a simple electrochemical route at room temperature. Structure analysis indicated that the $\mathrm{Ni}$ doped $\mathrm{ZnO}$ particles are polycrystalline of hexagonal wurtzite structure and the average size of the particles was estimated to be approximately $61 \mathrm{~nm}$, which conforms the nanoparticles in form. The band edge is shifted to the lower energy side of the nanoparticles. The FTIR result shows that the stretching vibration of the $\mathrm{H}-\mathrm{O}$ and $\mathrm{Zn}-\mathrm{O}$ bond. Photoluminescence spectrum measurement demonstrated that the nanoparticles exhibit a strong near band edge UV emission peak centred at $384 \mathrm{~nm}$.

\section{Acknowledgements}

We are grateful to Ministry of Science and Information, Communication Technology authority for financial support. We are thankful to Dr. M. Monjarul Alam, Mr. M. Abdul Momin for their assistance in preparing and measurement the samples.

\section{References}

[1] Xia B, Lenggoro W, Okuyama K. Novel route to nanoparticle synthesis by salt-assisted aerosol decomposition. Adv Mater 2001; 13: 1579-82.

[2] Lakshmi BB, Dorhout KP, Martin RC. Sol-gel template synthesis of semiconductor nanostructures. Chem Mater 1997; 9:857-62.

[3] Kobayashi S, Hanabusa K, Suzuki M, Kimura M, Shirai H. Preparation of TiO2 fiber in a sol-gel system containing organogelator. Chem Lett 1999; 10:1077-78.

[4] Kobayashi S, Hanabusa K, Hamasaki N, Kimura M, Shirai H, Shinkai S. Preparation of TiO2 hollowfibers using supramolecular assemblies. Chem Mater 2000; 12: 1523-25.

[5] Rao RNC, Satishkumar CB, Govindaraj A. Ziconia nanotubes.Chem Commun 1997; 16:1581-82.

[6] Satishkumar CB, Govindaraj A, Nath M, Rao NC. Synthesis of metal oxide nanorods using carbonnanotubes as templates.J Mater Chem 200; 10:2115-19.

[7] Li T, Qiu H, Wu P, Wang M, Ma R. Characteristics of Ni-doped ZnO:Al films grown on glass by direct current magnetron co-sputtering. Thin Solid Films 2007; 515:3905-09.

[8] Ueda K, Tabata H, Kawai T. Magnetic and electric properties of transition-metal-doped ZnO films. Appl Phys Lett 2001; 79:988-90.

[9] Wakano T, Fujimura N, Morinaga Y, Abe N, Ashida A, Ito T. Magnetic and magneto-transport properties of ZnO:Ni films. Physica E 2001; 10:260-64.

[10] Liu E, Xiao P, Chen JS, Lim BC, Li L. Ni doped ZnO thin films for diluted magnetic semiconductor materials. Current Appl Phys 2008; 8:408-11. 
[11] Pandey B, Ghosh S, Srivastava P, Avasthi DK, Kabiraj D, Pivin JC. Synthesis and characterization of Ni doped ZnO: A transparent magnetic semiconductor. J Mag Mag Mat 2008; 320:3347-51.

[12] Thota S, Kukreja LM, Kumar J. Ferromagnetic ordering in pulsed laser deposited Zn1 - xNixO/ZnO bilayer thin films. Thin Solid Films 2008; 517:750-54.

[13] Reetz MT, Helbig W. Size-selective synthesis of nanostructured transition metal clusters. J Am Chem Soc1994; 116:7401-02.

[14] Oh BY, Jeong MC, Kim DS, Lee W, Myoung JM. Post-annealing of Al-doped ZnO films in hydrogen atmosphere. J Cryst Growth 2005; 281:475-80.

[15] Mahmoud WE, Al-Ghamdi AA, El-Tantawy F, Al-Heniti S. Synthesis, characterization and charge transport mechanism of CdZnO nanorods. J Alloys Compd 2009; 485:59-63.

[16] Al-Harbi T. Hydrothermal synthesis and optical properties of $\mathrm{Ni}$ doped $\mathrm{ZnO}$ hexagonal nanodiscs. J Alloys Compd 2011; 509:387-90.

[17] Cong CJ, Hong JH, Liu QY, Liao L, Zhang KL. Synthesis, structure and ferromagnetic properties of Nidoped ZnO nanoparticles. Solid State Communications 2005; 138:511-15.

[18] Radovanovic PV, Gamelin DR. High-temperature ferromagnetism in Ni 2+ doped ZnO aggregates prepared from colloidal diluted magnetic semiconductor quantum dots. Phys Rev Lett 2003; 91:157202205.

[19] Wang Y, Liao X, Huang Z, Yin G, Gu J, Yao Y. Preparation and characterization of Ni-doped ZnO particles via a bioassisted process. Coll and Surf A: Physchem Engg Aspects 2010; 372:165-71.

[20] Kong YC, Yu DP, Zhang B, Fang W, Feng SQ. Ultraviolet-emitting ZnO nanowires synthesized by a physical vapor deposition approach. Appl Phys Lett 2001; 78:407-09.

[21] Yang JH, Wang DD, Yang LL, Zhang YJ, Xing GZ, Lang JH, Fan HG, Gao M, Wang Y. Effects of supply time of Ar gas current on structural properties of Au-catalyzed $\mathrm{ZnO}$ nanowires on silicon $\left(\begin{array}{lll}1 & 0 & 0\end{array}\right)$ grown by vapor-liquid-solid process. J Alloys Compd 2008; 450:508-11.

[22] Vanheusden K, Seager CH, Warren WL, Tallant DR, Voigt JA. Correlation between photoluminescence and oxygen vacancies in ZnO phosphors. Appl Phys Lett 1996; 68:403-05.

[23] Vanheusden K, Warren WL, Seager CH, Tallant DR, Voigt JA, Gnade BE. Mechanisms behind green photoluminescence in ZnO phosphor powders. J Appl Phys 1996; 79:7983-90.

[24] Schwartz DA, Kittilatved KR, Gamelin DR. Above-room-temperature ferromagnetic Ni 2+ doped ZnO thin films prepared from colloidal diluted magnetic semiconductor quantum dots. Appl Phys Lett 2004; 85:1395-97.

\section{Author(s) Profile}

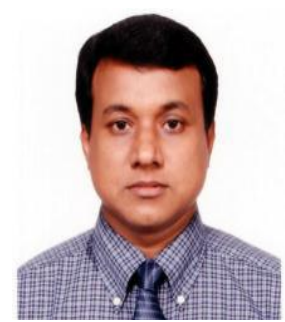

Prof. M. Ruhul Amin Bhyiyan was born on Dec.10, 1972 in Comilla, Bangladesh. He received the M.Sc degree in Applied Physics and Electronics from Rajshahi University, Bangladesh in 1995, and Ph.D. degree from Islamic University, Kushtia, Bangladesh in 2008. He is currently a Professor in the Department of APE \& Com. Engineering, Islamic University, Bangladesh. His current interest is thin-film solar cell and nanoparticle materials characterization. His work has produced nearly 30 peer-reviewed scientific International and National papers. 
Md. Khalilur Rahman was born on July 1, 1971 in Madaripur, Bangladesh. He received the M.Sc. degree in Applied Physics, Electronics \& Communication Engineering from University of Dhaka, Bangladesh in 1992. He is currently an Associate Professor in the Department of Applied Physics, Electronics \& Communication Engineering, Faculty of Applied Science \& Technology, Islamic University, Kushtia7003, Bangladesh. His interest is in Optoelectronics and Wireless Communication. His work has produced nearly 20 National and International papers.

How to cite this paper: M.R.A. Bhuiyan, M.K. Rahman,"Synthesis and Characterization of Ni Doped ZnO Nanoparticles", IJEM, vol.4, no.1, pp.10-17, 2014.DOI: 10.5815/ijem.2014.01.02 